In Sendai, Japan 2000 SPIE Meeting Proceedings

\title{
Obtaining Calibrated Marine Aerosol Extinction Measurements Using Horizontal Lidar Measurements, Differential Lidar-Target Measurements and A Polar Nephelometer.
}

\author{
J. N. Porter, S. K. Sharma, and B. R. Lienert \\ University of Hawaii \\ School of Ocean and Earth Science and Technology \\ Hawaii Institute of Geophysics and Planetology \\ Honolulu, Hawaii
}

\begin{abstract}
Lidars are ideal for mapping the spatial distribution of aerosol concentrations, however efforts to convert the lidar measurements into estimates of the aerosol extinction or scattering coefficient are usually complicated. The difficulties arise from the uncertainty in the aerosol backscatter-to-extinction ratio and the lidar calibration. In marine conditions with little absorption, the aerosol backscatter-to-extinction ratio is identical to the aerosol phase function $/ 4 \pi$ at 180 degrees (backscatter). Uncertainty in the lidar calibration is another source of uncertainty, which can change with time depending on the state of the optics (clean or dirty). Here we investigate several techniques to obtain calibrated aerosol extinction coefficient values. The first approach uses horizontal lidar measurements over the open ocean where the atmosphere is horizontally homogeneous. The lidar calibration or aerosol phase function is adjusted until the derived aerosol extinction coefficients are flat with distance. Modeling shows that this provides correct aerosol extinction values. A second approach uses a target to reflect the lidar beam at different distances. The aerosol extinction is derived from the differential transmission measurements. As an independent measurement, the aerosol phase function and scattering coefficients can be measured with a polar nephelometer.
\end{abstract}

Keywords: Lidar calibration, aerosol extinction, aerosol phase function, target calibration

\section{INTRODUCTION}

For a mono-static lidar systems the lidar equation can be written as

$$
\begin{aligned}
& n(r)=\left(\frac{\lambda \delta_{\lambda}}{h c} \Delta r E_{o}\right)\left(\frac{A_{r}}{r^{2}}\right) \beta(r) T(r)^{2} \\
& \text { alternatively } \\
& n(r)=C\left(\frac{A_{r}}{r^{2}}\right)\left(\frac{\bar{P}_{180}(r)}{4 \pi}\right) \sigma_{s}(r) T(r)^{2}
\end{aligned}
$$

where $n(r)$ is the photoelectrons (signal) received from distance $r$ and range bin $\Delta r . E_{\mathrm{o}}$ is the laser pulse energy at wavelength $\lambda$. The detector quantum efficiency is $\delta_{\lambda}, h$ is Planck's constant, $c$ is the speed of light, $A$ is the telescope effective area, and $\beta(r)$ is the back scattering coefficient at range $r$. In the second form of Eq. 1,C is an overall lidar calibration coefficient, $\sigma_{\mathrm{s}}(\mathrm{r})$ is the total scattering coefficient at range $\mathrm{r}$, and $\bar{P}_{180}(r)$ is the weighted phase function (for 180 degree scattering angle) at range r. $T(r)^{2}$ is the atmospheric transmission term to range $\mathrm{r}$ and is given by 


$$
T(r)^{2}=e^{-2 \int \sigma_{e}(r) d r}
$$

(2)

The extinction coefficient $\left(\sigma_{\mathrm{e}}(r)\right)$ is the sum of the scattering and absorption coefficients $\left(\sigma_{\mathrm{s}}(r)+\sigma_{\mathrm{a}}(r)\right)$. The scattering coefficient, $\sigma_{\mathrm{s}}(r)$, is the sum of the aerosol and molecular scattering coefficients $\left(\sigma_{\mathrm{as}}(r)+\sigma_{\mathrm{ms}}(r)\right)$. The absorption coefficient is also due to aerosol absorption and molecular absorption $\left(\sigma_{\text {aa }}(\mathbf{r})+\sigma_{\mathrm{ma}}(r)\right)$. The molecular absorption coefficient $\left(\sigma_{\mathrm{ma}}(r)\right)$ is often negligible because lidar wavelengths are chosen where little atmospheric absorption occurs. The aerosol phase function in Eq. 1 is the weighted average of the aerosol and molecular phase functions given by

$$
\bar{P}_{180}(r)=\frac{P_{m 180}(r) \sigma_{m s}(r)+P_{a 180}(r) \sigma_{a s}(r)}{\sigma_{m s}+\sigma_{a s}}
$$

(3)

where $P_{m 180}(r)$ is the molecular phase function at range r, $P_{a 180}(r)$ is the aerosol phase function at range r, $\sigma_{m s}(r)$ is the molecular scattering coefficient at range $\mathrm{r}, \sigma_{a s}(r)$ is the aerosol scattering coefficient at range $\mathrm{r}$. In using Eq. 1-3 we would like to derive the aerosol extinction coefficients.

Unfortunately both the aerosol phase function and lidar calibration are also typically not known. Due to these difficulties, numerous inversion techniques have been proposed. As summarized by Reagan et al. (1989), these include the slope method (Collis, 1966), modeled transmission method (Russell et al., 1979), slant path method (Sandford, 1967), and various methods using a relationship between extinction and backscatter (Hitschfeld and Bordan, 1954, Fernald et al., 1972, Klett, 1981). Klett also proposed using a known value of the aerosol scattering at a distant point and solving for the along-path extinction with a backward stepping approach, which he showed to be more stable than the forward stepping approach. This approach was found to be very sensitive to the lidar calibration and the power law relation between the backscattering coefficient and the extinction coefficient (Hughes et al., 1985). If the aerosols are horizontally homogeneous then horizontal or slant measurements can be used to provide boundary values to constrain the aerosol extinction coefficients (Zhang and $\mathrm{Hu}, 1997$ ) and even to estimate the aerosol phase function (Young et al., 1993). Porter et al. (2000) used a forward stepping approach to derive the aerosol scattering coefficient for open ocean marine air. In this paper we will expand on the Porter et al. approach to include absorbing aerosol and discuss several other methods.

\section{FORWARD STEPPING ALGORITHM}

The Porter et al. (2000) forward stepping method of deriving aerosol scattering coefficients is described. The forward stepping lidar inversion begins by assuming a fixed aerosol transmission, $T_{\mathrm{a}}(\mathrm{r})$, from the lidar out to range, $r \sim 300 \mathrm{~m}$ to step past the near field region where the scattered light cannot be properly focused on the detector. The molecular transmission, $T_{\mathrm{m}}(\mathrm{r})$, from the lidar out to the same starting point is calculated based on pressure and temperature. Beyond this near-field region the inversion is begun by calculating the aerosol scattering coefficient in the next sample bin at distance $\mathrm{r}(r-\Delta r / 2$ to $r+\Delta r / 2)$ using

$$
\sigma_{a s}(r)=\left(n(r) \frac{\left(4 \pi r^{2}\right)}{C\left(T_{m}(r-\mathrm{V} r) T_{a}(r-\mathrm{V} r)\right)^{2}}-P_{m} \sigma_{m s}\right) \frac{1}{P_{a}}
$$

where equations (1)-(3) have been combined and solved for the aerosol scattering coefficient. The aerosol transmission (due to absorption and scattering) from the lidar to the bin is given by $T_{a}(r)=T_{a}(r-\Delta r) \Delta T_{a}(r) . \mathrm{V} T_{a}(r)=e^{-\sigma_{a s}(r) \mathrm{V} r}$ is calculated using the aerosol extinction calculated in eq. (4). Similarly a new molecular transmission, is calculated by $T_{m}(r)=T_{m}(r-\Delta r) \Delta T_{m}(r)$ where $\mathrm{V} T_{m}(r)=e^{-\sigma_{m s}(r) \mathrm{V} r}$. The molecular scattering coefficient is computed at the proper pressure and temperature following Lenoble (1993) using the hypsometric equation for the height dependence. Next a 
new aerosol scattering coefficient is calculated in the next sample bin using eq. (5) again. The process is repeated out to the end of the lidar measurement. A schematic of the forward stepping approach is given in Fig. 1.

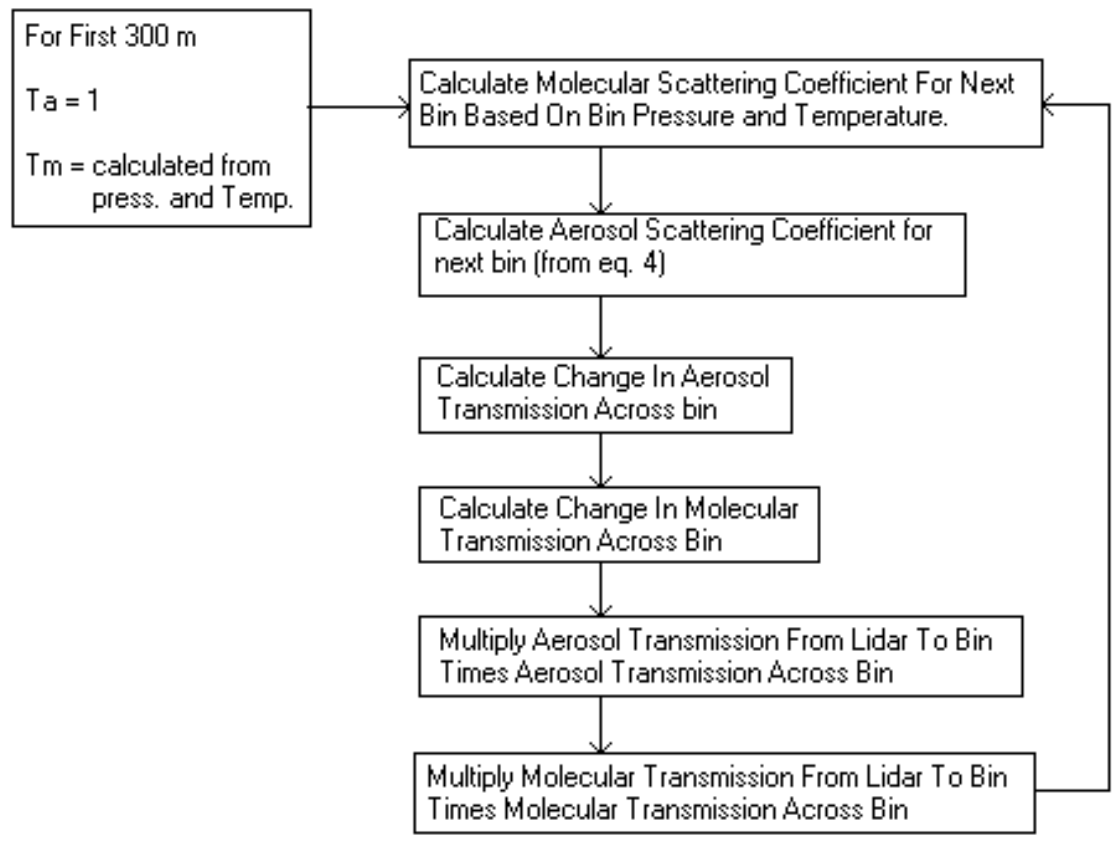

Figure 1. Chart of forward stepping algorithm.

This forward stepping approach has no restriction on the decreasing transmission and can accumulate large error at longer distances. The error comes from using an incorrect aerosol phase function (at 180 degrees) or wrong lidar calibration (Hughes et al., 1985). Here it is assumed that the lidar is properly aligned and the laser beam is within the collection cone of the receiver optics.

\section{MODELING TESTS}

In order to test this forward stepping approach, we generate pseudo-lidar data by using eq. (1)-(3). For the model lidar data, the aerosol phase function (phase function at 180 degrees) value was set to 0.65 , to represent marine sea salt. The aerosol scattering coefficient was set to $5 \times 10^{-5} \mathrm{~m}^{-1}$. The aerosol absorption coefficient was set to 0 . The aerosol is assumed to be horizontally homogeneous. The lidar calibration $C$ is set to a fixed value. The molecular scattering coefficient was set to $1.211 \times 10^{-5} \mathrm{~m}^{-1}$ corresponding to surface pressure $(1013 \mathrm{mb})$ and $532 \mathrm{~nm}$ wavelength. Figure 2 shows the modeled lidar return signal from a horizontal lidar measurement with homogeneous aerosol. The lidar signal has been plotted versus the aerosol optical thickness and the distance is shown on the right side. The relationship between the lidar signal and the $1 / r^{2}$ term (scaled to fit on the same plot) is also shown as a function of optical thickness. Noise has also been introduced to simulate digitization and electronic noise.

Using the forward stepping approach described above, the aerosol scattering coefficient is derived from the modelgenerated data using correct and incorrect values of the aerosol phase function (at 180 degree). These results are shown in Fig. 3, where it can be seen that the error increases with distance when wrong values of the phase function are used. As expected, using the correct aerosol phase function value, yields the correct aerosol scattering coefficient $\left(5 \times 10^{-5} \mathrm{~m}^{-1}\right)$ at all distances. Greater error results if the aerosol phase function is underestimated than if overestimated. If the aerosol phase function is overestimated the drop-off with range is not so strong and might appear as a straight line if measurements are not made to large enough optical depths. Using incorrect values for the lidar calibration value $(C)$ cause similar errors to those shown in Figure 3. In fact, incorrect lidar calibration and incorrect aerosol phase function values can be combined to produce derived extinction values which are constant with range and have the correct 
extinction value.

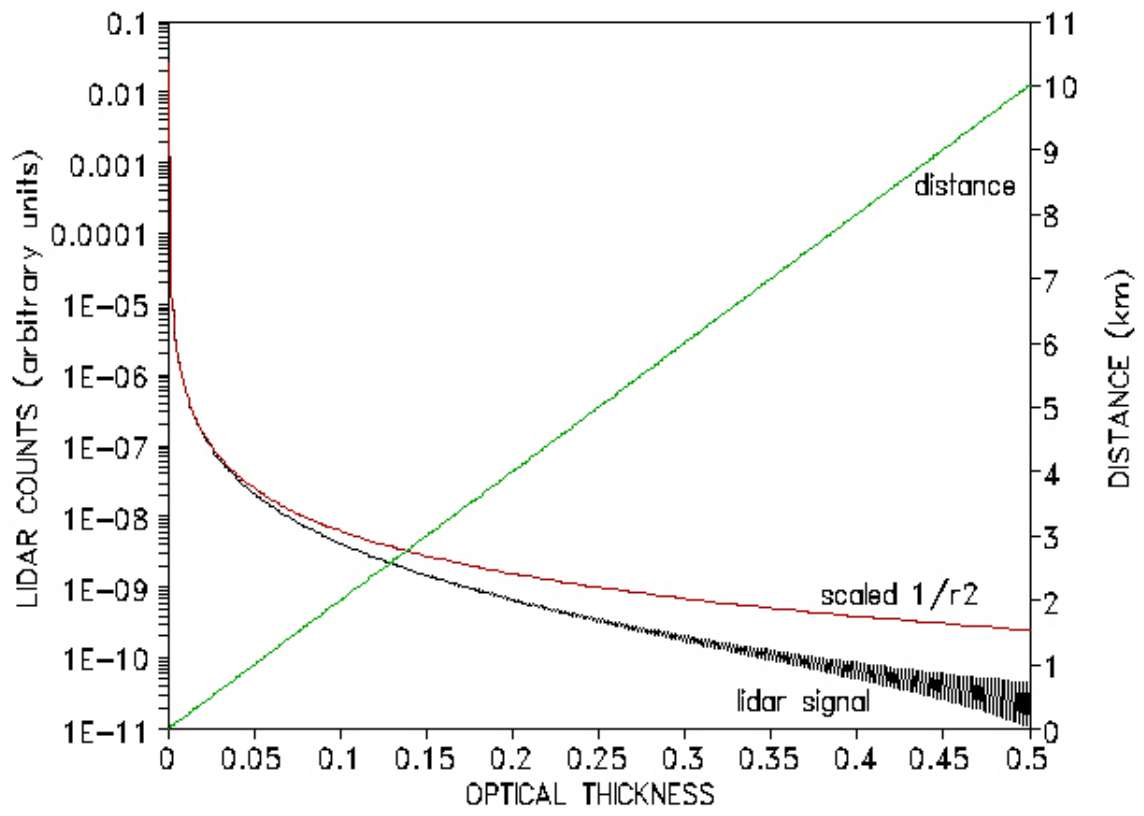

Figure 2. Pseudo lidar signal generated from Eq. 1 as a function of aerosol optical depth.

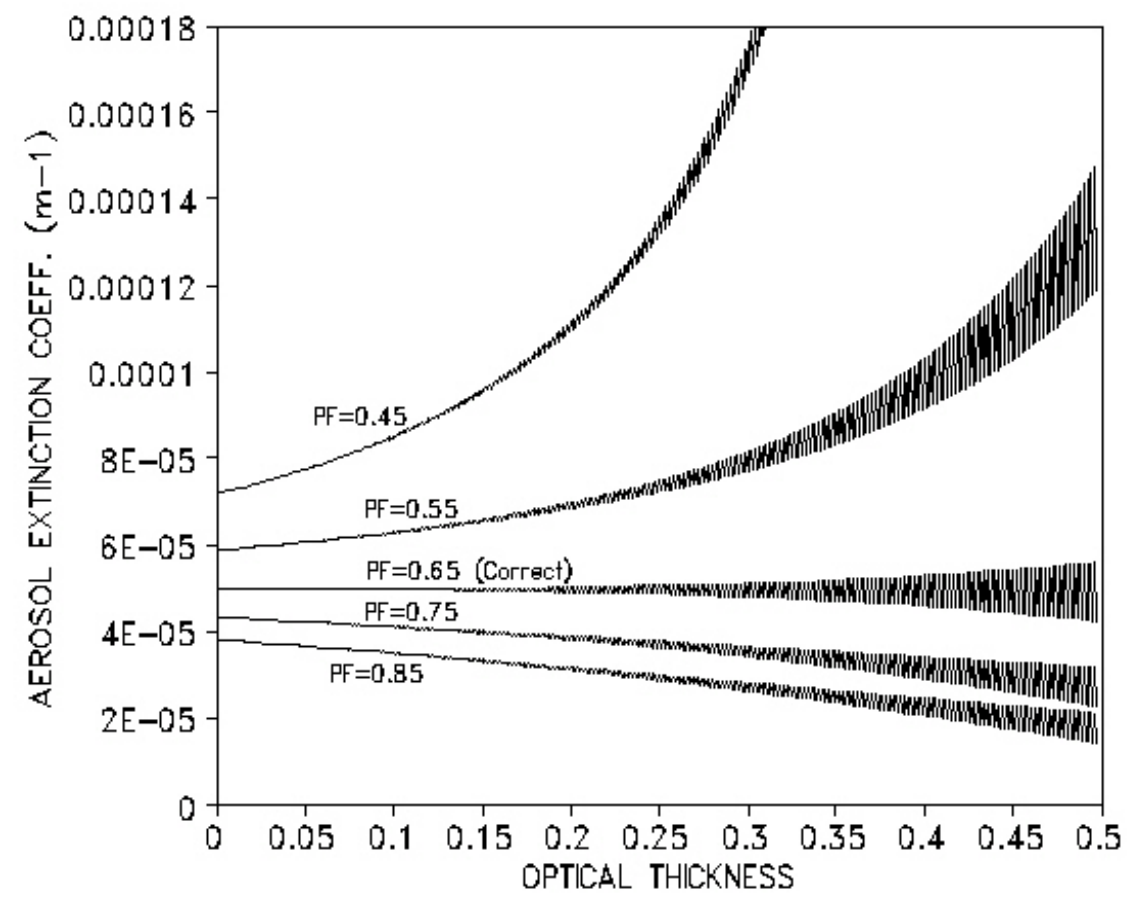

Figure 3. Aerosol extinction coefficient derived from forward stepping approach.

The calculations done above have assumed no aerosol absorption. Figure 4 shows the results when the aerosol absorption is included in the generation of the lidar data. For these test the aerosol scattering and absorption coefficient values were set to $5 \times 10^{-5} \mathrm{~m}^{-1}$ (aerosol single scatter albedo of 0.5 ). In the inversion the aerosol were assumed to be only 
scattering with no absorption. It can be seen that the derived values are not constant with range and are less than the correct value. If the aerosol phase function is manually adjusted so that the derived scattering coefficients are constant with range (Fig. 5)

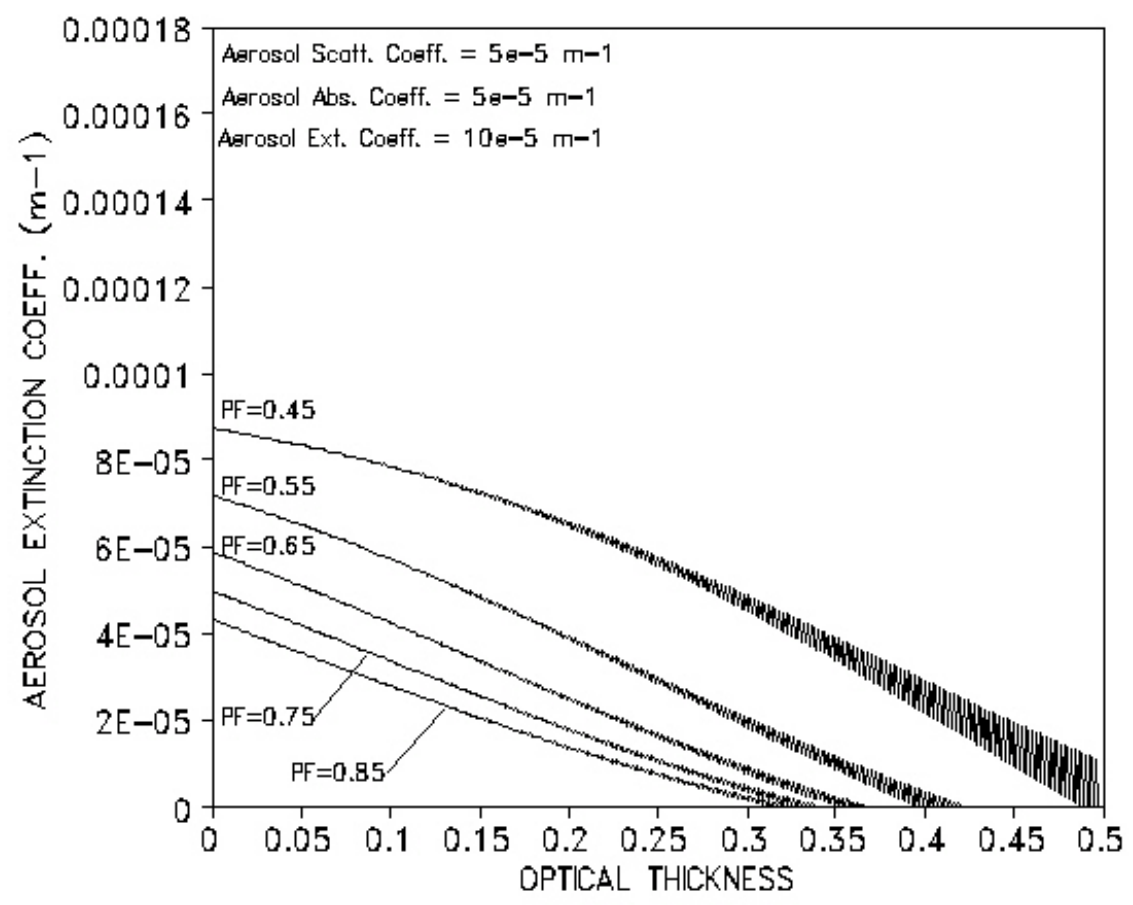

Figure 4 Aerosol extinction coefficients derived from forward stepping approach.

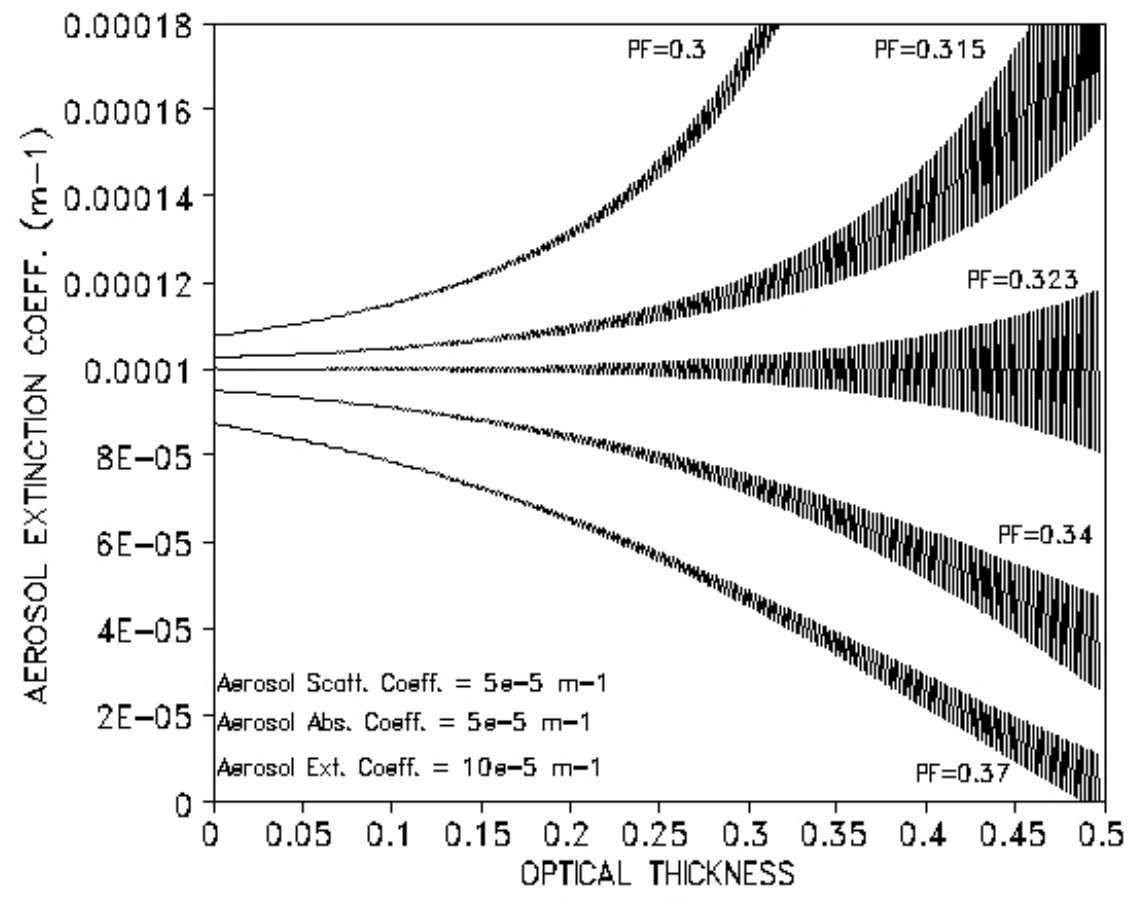

Figure 5. Aerosol extinction coefficients derived from forward stepping approach. 
then the derived extinction values are correct $\left(10 \mathrm{e}^{-5} \mathrm{~m}^{-1}\right)$ but an incorrect aerosol phase function value $(0.323)$ must be used.

When aerosol absorption is present in the lidar data and is not used in the forward stepping inversion (as Fig. 5), the ratio of the aerosol single scatter albedo is equal to the ratio of the phase function required to obtain constant extinction values with distance over the correct phase function. From the examples shown in Figs. 3-5, it can be seen that varying either the aerosol phase or the lidar calibration (Porter et al., 2000) can adjust the derived aerosol extinction values so that they are constant with range. When this is done the resulting extinction values are correct for both absorbing and non-absorbing aerosol.

\section{TARGET CALIBRATION APPROACH}

Equation 1 can be rearrange into the following equation

$$
\ln \left(r^{2} n(r)\right)=-2 \sigma_{e} r+\ln \left(C A_{r} \beta(r)\right) .
$$

Equation 5 has the form of a straight line and can be plotted with $\ln \left(r^{2} n(r)\right)$ (y values) plotted versus $\mathrm{r}$ (x values).

The slope of the data is then $-2 \sigma_{e}$, and the zero intercept is $\ln \left(C A_{r} \beta(r)\right)$ (Reagan, 1995). While this approach is excellent for deriving the extinction coefficient $\left(\sigma_{e}\right)$, it is difficult to derive the lidar calibration, $\mathrm{C}$, because the backscattering coefficient, $\beta(r)$, is often unknown due to uncertainty in the aerosol properties. As a modification to this approach, we have begun testing a new method to calibrate our lidar using a moving target and measuring the return pulse from the target. In this case, the backscattering coefficient is due to the properties of the target, which is presumably well known. Therefore, the lidar calibration can be derived by fitting a line to the data and extrapolating the line back to the zero intercept.

Figure 6. Lidar signal measured from a dark Spectralon target at different distances.

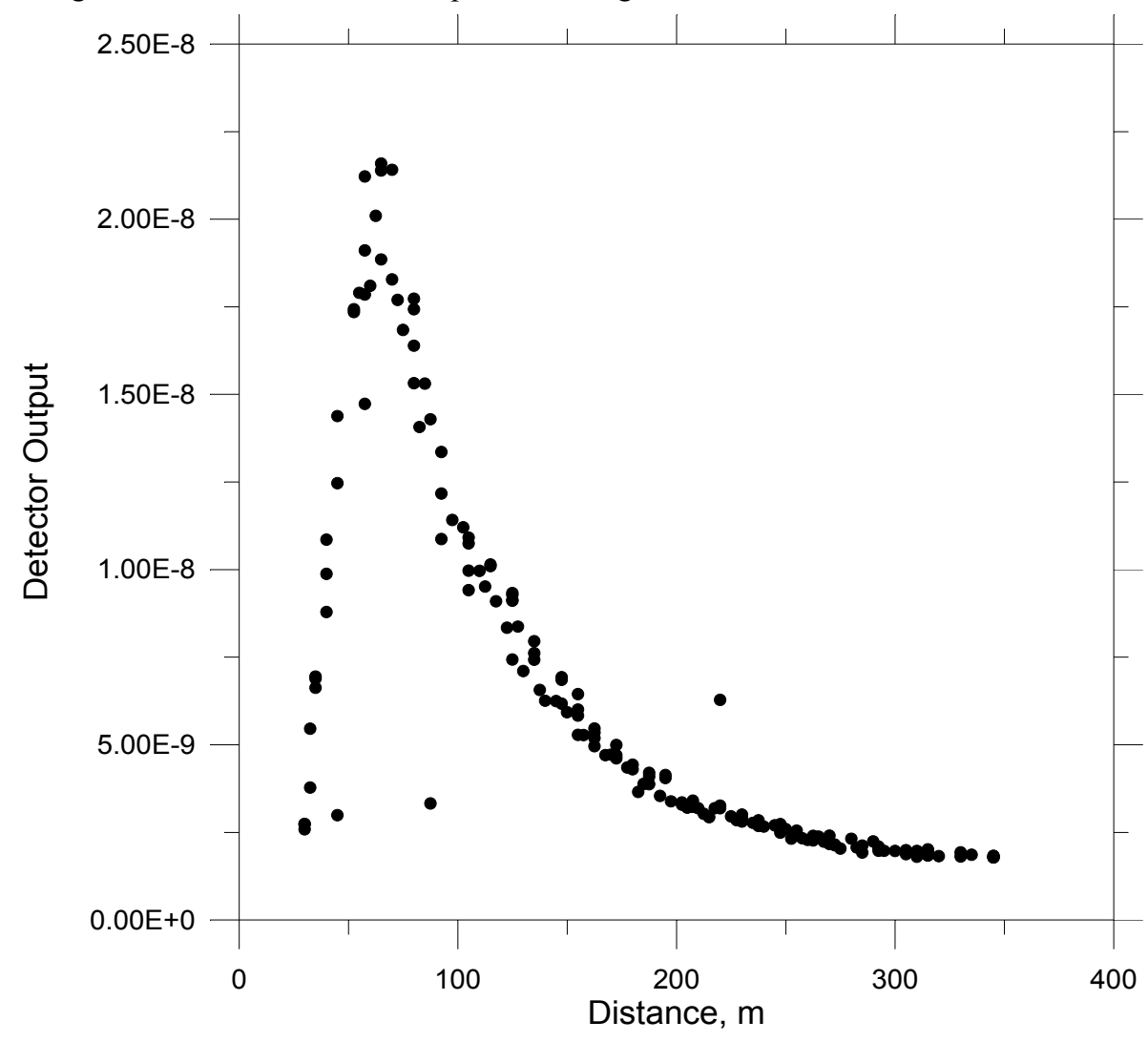

Figure 6 shows our preliminary lidar measurements made using a dark spectralon target at different distances. It can be 
seen that the signal is strongly attenuated in the near field. This near field effect is expected due to the central obstruction in our Schmidt-Cassegrain mono-static lidar (Sharma et. al., 1998; Lienert, et al., 1999). Past approximately $75 \mathrm{~m}$ the target signal appears to drops off with roughly a $1 / \mathrm{r}^{2}$ dependence but continues to be affected by the near field effect as well. Based on modeling studies our lidar system we estimate that the target measurements need to be made at a distance greater than $300 \mathrm{~m}$ to avoid any significant near field effects. We will be carrying out additional test with this promising approach.

\section{POLAR NEPHELOMETER METHOD}

For non-absorbing aerosol, an additional way to test our lidar measurements is to measure the aerosol phase function and scattering coefficient. Doherty et al. (1989) have made measurements of the aerosol phase function at backscattering angles in a closed system. We are currently developing two open-air polar nephelometers, which will measure the aerosol phase function and scattering coefficient. These systems are improvements over our earlier CW system (Porter et al., 1997). The new systems use an Nd-YAG pulsed laser with light at 1064, 532 and $355 \mathrm{~nm}$. Either a PMT or APD detector is used depending on wavelength. Angular measurements are made every 1-degree (2-178 degrees) with our larger ground based system and every 6 degrees (6-174 degrees) with our aircraft system. Figure 7 shows some measurements made a Bellows Beach in Hawaii. These test measurements were made before the system was complete and include both polarization components of polarized laser light scattered from the aerosol. Both systems are currently under final modifications and we expect to begin making measurements soon.

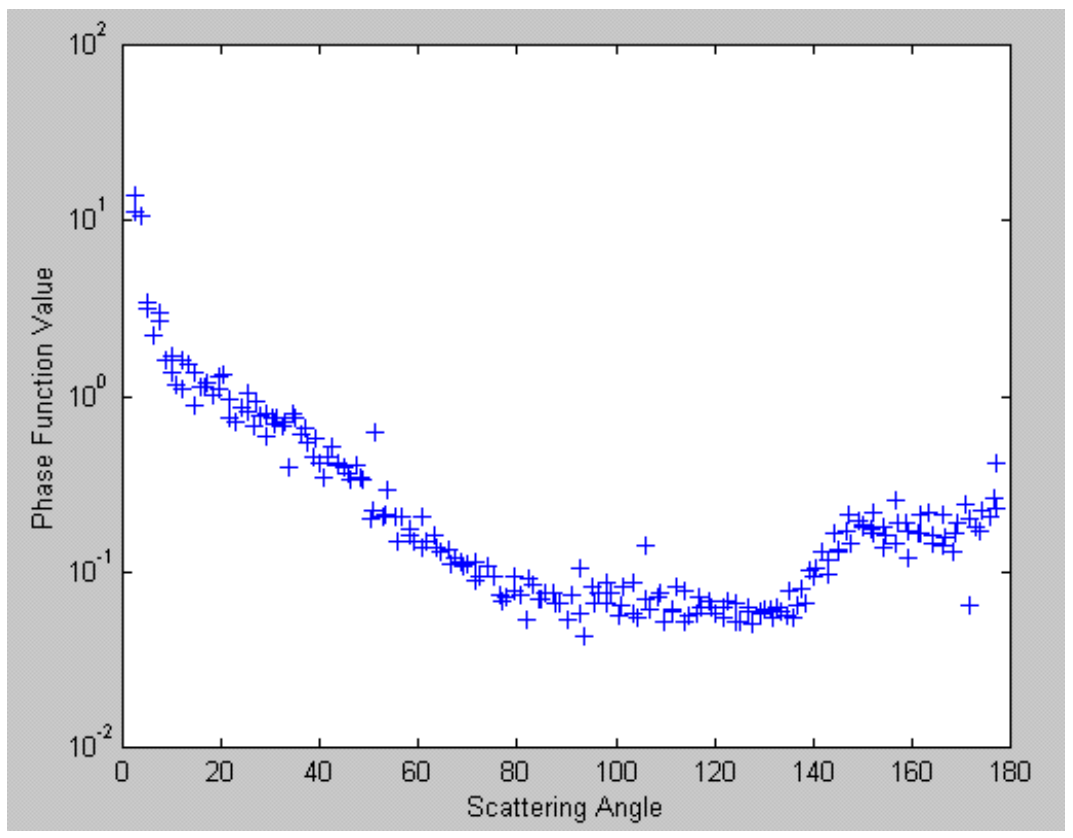

Figure 7. Aerosol phase function measurements at a coastal site.

\section{CONCLUSION}

While calibration of Mie-Rayleigh lidars is challenging, various techniques do exist to convert the measurements into standard optical values such as extinction and scattering coefficients. We have found that the horizontal method is very useful and easy to implement and can give accuracies better than $25 \%$. Although the target method has promise, it requires measurements over a long distance to avoid near field effects on the lidar calibration. Finally, the addition of new instruments such as the open air polar nephelometers will provide important validation data sets such the aerosol phase function and aerosol scattering coefficient near the lidar site. 


\section{ACKNOWLEDGEMENTS}

We would like to thank Steve Ackleson and Ron Ferek for their support. The work carried out here was funded by an ONR grant \# N00014-96-1-0317.

\section{REFERENCES}

Collis, R.T. H., 1966: Lidar: a new atmospheric probe. Quart. J.Roy. Meteorol. Soc., vol. 92, pp.220-230.

Doherty, S.J., T. L. Anderson, and R.J. Charlson, 1999: Measurement of the lidar ratio for atmospheric aerosols with a $180^{\circ}$ backscatter nephelometer. Appl. Opt., vol. 38, pp 1823-1832.

Fernald, F.G., B.M. Herman, and J.A. Reagan, 1972: Determination of aerosol height distribution by lidar. J. Appl. Meteorol., vol. 11, pp. 482-489.

Hitchfeld, W., and J. Bordan, 1954: Errors inherent in the radar measurements of rainfall at attenuating wavelengths. J. Meteorol., vol. 11, pp 58-67.

Huges, H.G., J.A. Ferguson, and D.H. Stephens, 1985: Sensitivity of lidar inversion algorithm to parameters relating atmospheric backscatter and extinction. Appl. Opt. Vol. 24, pp. 1609-1613.

Klett, J.D., 1981; Stable analytical inversion solution for processing lidar returns. Applied Optics, vol.20, no. 2, pp. 211 220.

Lienert, B.R., J. N. Porter, and S.K. Sharma, 1999; Real time analysis and display of scanning lidar scattering data. Marine Geodesy, vol. 22, pp. 259-265.

Porter, J.N., B. Lienert, S.K. Sharma, 2000: Using Horizontal and Slant Lidar Measurements To Obtain Calibrated Aerosol Scattering Coefficients From A Coastal Lidar In Hawaii, accepted in J. Atmospheric and Oceanic Technology.

Porter, J.N., T.F. Cooney, C. Motell, 2000: Coastal Aerosol Phase Function Measurements With A Custom Polar Nephelometer, Ocean Optics XIV Conference, Kona, Hawaii.

Reagan, J.A., 1995: New generation lidars to support aerosol/climate forcing studies. IEEE, IGARS95, Florence, Italy, July 10-14, catalog \# 95CH35770, pp 2313-2315.

Reagan, J.A, P. M.P. McCormick, and J.D. Spinhirne, 1989: Lidar sensing of aerosols and clouds in the troposphere and stratosphere, Proceedings of the IEEE, vol. 77, no. 3, pp 433-448.

Russell, P.B., T.J. Swissler, and M. P. McCormick, 1979: Methodology for error analysis and simulation of lidar aerosol measurements. Appl. Opt., vol. 18, pp. 3783-3797.

Sandford, M.C.W., 1967: Laser scatter measurements in the mesosphere and above. J. Atmos. Terr. Phys., vol. 29, pp 1657-1662.

Sharma, S.K., B. R. Lienert, J. N. Porter, A. D. Clarke, and J. Howell, Scanning lidar imaging of marine aerosol fields generated by breaking waves. In U. N. Singh, S. Ismail, and G. K. Schwemmer (eds.) Proceedings of the $19^{\text {th }}$

International Laser Radar Conference, Part 2, pp. 673-676, NASA Langley Research Center, Hampton, Virginia, USA, 1998. 
Young, S. A., D. R. Cutten, M. J. Lynch and J. E. Davies, 1993: Lidar-derived variations in the backscatter-to-extinction ratio in southern hemisphere coastal maritime aerosols. Atmospheric Environment, vol. 27, no. 10, pp 1541-1555.

Zhang, J., and H. Hu, 1997: Lidar calibration: A new method. Applied Optics, Vol. 36, no.6, pg 1235-1238. 